\title{
Focused labeled proof systems for modal logic
}

\author{
Dale Miller and Marco Volpe \\ Inria and LIX, École Polytechnique, France
}

\begin{abstract}
Focused proofs are sequent calculus proofs that group inference rules into alternating positive and negative phases. These phases can then be used to define macro-level inference rules from Gentzen's original and tiny introduction and structural rules. We show here that the inference rules of labeled proof systems for modal logics can similarly be described as pairs of such phases within the $L K F$ focused proof system for first-order classical logic. We consider the system G3K of Negri for the modal logic $K$ and define a translation from labeled modal formulas into first-order polarized formulas and show a strict correspondence between derivations in the two systems, i.e., each rule application in G3K corresponds to a bipole - a pair of a positive and a negative phases - in LKF. Since geometric axioms (when properly polarized) induce bipoles, this strong correspondence holds for all modal logics whose Kripke frames are characterized by geometric properties. We extend these results to present a focused labeled proof system for this same class of modal logics and show its soundness and completeness. The resulting proof system allows one to define a rich set of normal forms of modal logic proofs.
\end{abstract}

\section{Introduction}

What is an inference rule? If we try to answer this question in the setting of the sequent calculus, then it seems that we should ask that inference rules have duals and that all occurrences of cut rules and non-atomic initial rules can be eliminated. In a two-sided sequent system, dual inference rules are typically pairs of left and right introduction rules for a given connective. In a one-side sequent system, dual inference rules are usually based on introduction rules for de Morgan dual connectives. Such a definition of inference rules has been suggested by Girard in [9, Section F.5] and formalized by Miller and Pimentel in [13].

In recent years, focused proof systems have been introduced as a means of building large scale synthetic inference rules from Gentzen's original, small scale introduction rules. In particular, Andreoli introduced a focused proof system for linear logic [1] and described cut-free proofs as alternating phases of inference rules: a negative phase is a collection of invertible inference rules and a positive phase is a collection of inference rules that are dual to those in negative phases. This same kind of focused proof system has also been extended to both intuitionistic and classical logic in the $L J F$ and $L K F$ proof systems [10]: the $L K F$ proof system will play a central role in this paper. In all of these focused proof systems, phases can be used to describe synthetic inference rules by identifying them with either an entire positive or negative phase. In all these cases, cuts and non-atomic initial rules can be eliminated at the level of synthetic inference rules. 
In this paper, we look at focused proof systems and their possible relationship to modal logic proof systems based on labeled sequents. We shall show that it is possible to emulate precisely the G3K proof system [14] using a simple encoding of modal formulas and inference rules into classical first-order logic in such a way that one inference rule of $G 3 K$ exactly corresponds to one phase in the translated logic. Such tight emulation means that if one does proof search or proof checking on the focused version of the translated formulas, one is modeling nothing more or less than proof search in G3K.

One alternation of a positive followed by a negative phase (reading from conclusion to premises) is a natural unit of inference in a focused proof system: such a pair of phases is called a bipole. A formula that induces a bipole is a bipolar formula and examples of such formulas are geometric formulas, when properly polarized. As a result, we are able to show that we can use focused classical proofs to precisely emulate modal proofs whenever Kripke frames are characterized by geometric properties. Since every (infinitary) first-order theory has a geometric conservative extension [6], the limitation to geometric theories is not restrictive. We also present a focused proof system for any classical propositional modal logic whose Kripke frames are described using geometric theories.

\section{Background}

\subsection{Modal logic}

The language of (propositional) modal formulas consists of a functionally complete set of classical connectives (here we will use a minimal one, but other connectives, defined as usual, will be sometimes employed in the rest of the paper), a modal operator $\square$ (here we will also use explicitly its dual $\diamond$ ) and a denumerable set $\mathcal{P}$ of propositional symbols, according to the following grammar:

$$
A::=P|\perp| A \supset A|\square A| \diamond A,
$$

where $P \in \mathcal{P}$. The semantics is usually defined by means of Kripke frames, i.e., pairs $\mathcal{F}=(W, R)$ where $W$ is a non empty set of worlds and $R$ is a binary relation on $W$. A Kripke model is a triple $\mathcal{M}=(W, R, V)$ where $(W, R)$ is a Kripke frame and $V: W \rightarrow 2^{\mathcal{P}}$ is a function that assigns to each world in $W$ a (possibly empty) set of propositional symbols.

Truth of a modal formula at a point $w$ in a Kripke structure $\mathcal{M}=(W, R, V)$ is the smallest relation $\models$ satisfying:

$$
\begin{array}{rll}
\mathcal{M}, w \models P & \text { iff } & p \in V(w) \\
\mathcal{M}, w \models A \supset B & \text { iff } & \mathcal{M}, w \models A \text { implies } \mathcal{M}, w \models B \\
\mathcal{M}, w \models \square A & \text { iff } & \mathcal{M}, w^{\prime} \models A \text { for all } w^{\prime} \text { s.t. } w R w^{\prime} \\
\mathcal{M}, w \models \diamond A & \text { iff } & \text { there exists } w^{\prime} \text { s.t. } w R w^{\prime} \text { and } \mathcal{M}, w^{\prime} \models A .
\end{array}
$$

By extension, we write $\mathcal{M} \models A$ when $\mathcal{M}, w \models A$ for all $w \in W$ and we write $\models A$ when $\mathcal{M}=A$ for every Kripke structure $\mathcal{M}$. The former definition characterizes the basic modal logic $K$. Several further modal logics can be defined as extensions 


\begin{tabular}{|c|c|c|}
\hline Axiom & Condition & First-Order Formula \\
\hline $\mathrm{T}: \square A \supset A$ & Reflexivity & $\forall x . R(x, x)$ \\
\hline $4: \square A \supset \square \square A$ & Transitivity & $\forall x, y, z .(R(x, y) \wedge R(y, z)) \supset R(x, z)$ \\
\hline $5: \square A \supset \square \supset A$ & Euclideaness & $\forall x, y, z .(R(x, y) \wedge R(x, z)) \supset R(y, z)$ \\
\hline $\mathrm{B}: A \supset \square \diamond A$ & Symmetry & $\forall x, y \cdot R(x, y) \supset R(y, x)$ \\
\hline 3: $\square(\square A \supset B) \vee \square(\square B \supset A)$ & Connectedness & $\forall x, y, z .(R(x, y) \wedge R(x, z)) \supset(R(y, z) \vee R(z, y))$ \\
\hline $\mathrm{D}: \square A \supset \diamond A$ & Seriality & $\forall x \exists y \cdot R(x, y)$ \\
\hline $2: \diamond \square A \supset \square \diamond A$ & Directedness & $\forall x, y, z .(R(x, y) \wedge R(x, z)) \supset \exists t(R(y, t) \wedge R(z, t))$ \\
\hline
\end{tabular}

Table 1. Axioms and corresponding first-order conditions on $R$.

of $K$ by simply restricting the class of frames we consider. Many of the restrictions we are interested in are definable as formulas of first-order logic where the binary predicate $R(x, y)$ refers to the corresponding accessibility relation. Table 1 summarizes some of the most common frame logics, describing the corresponding frame property, together with the modal axiom capturing it [17]. We will refer to the logic satisfying the axioms $F_{1}, \ldots, F_{n}$ as $K F_{1} \ldots F_{n}$.

\subsection{A labeled proof system for modal logic}

The basic idea behind labeled proof systems for modal logic is to internalize elements of the corresponding Kripke semantics (namely, the worlds of a Kripke structure and the accessibility relation between such worlds) into the syntax. As a concrete example of such a system, here we will consider the system $G 3 K$ presented in [14]. G3K formulas are either labeled formulas of the form $x: A$ or relational atoms of the form $x R y$, where $x, y$ range over a set of variables and $A$ is a modal formula (here we consider also $\wedge$ and $\vee$ as primitive connectives). In the following, we will use $\varphi, \psi$ to denote $G 3 K$ formulas. G3K sequents have the form $\Gamma \vdash \Delta$, where $\Gamma$ and $\Delta$ are multisets containing labeled formulas and relational atoms. In Figure 1, we present the rules of G3K, which is proved to be sound and complete for the basic modal logic $K$ [14]. The system is then extended to cover all modal logics whose Kripke frames are determined by geometric axioms (note that all the logics in Table 1 fall inside this class), i.e., axioms of the form:

$$
\forall \bar{z}\left(P_{1} \wedge \ldots \wedge P_{m} \supset\left(\exists x_{1}\left(Q_{11} \wedge \ldots \wedge Q_{1 k_{1}}\right) \vee \ldots \vee \exists x_{n}\left(Q_{n 1} \wedge \ldots \wedge Q_{n k_{n}}\right)\right)\right)
$$

where each $P_{i}$ and $Q_{j k}$ is a relational atom ${ }^{1}$. As described in [14], the following general rule scheme

$$
\frac{\overline{Q_{1}}\left(y_{1} / x_{1}\right), \bar{P}, \Gamma \vdash \Delta \quad \ldots \quad \overline{Q_{n}}\left(y_{n} / x_{n}\right), \bar{P}, \Gamma \vdash \Delta}{\bar{P}, \Gamma \vdash \Delta} G R S
$$

can be used instead of the geometric axiom above: here $\overline{Q_{j}}$ and $\bar{P}$ denote the multisets of relational atoms $Q_{j 1}, \ldots, Q_{j k_{j}}$ and $P_{1}, \ldots, P_{m}$, respectively, and the eigenvariables $y_{1}, \ldots, y_{n}$ do not occur free in the conclusion. In Figure 2, the rules for capturing the frame properties of Table 1 are shown. By modularly

\footnotetext{
${ }^{1}$ Note that, for simplicity, as in [14], we restrict to the case where only a single variable is bound to each existential quantifier.
} 
INITIAL RULES

$$
\overline{x: P, \Gamma \vdash \Delta, x: P} \text { init }
$$

Propositional RUles

ModAl RULES

$$
\begin{array}{cc}
\frac{x: A, x: B, \Gamma \vdash \Delta}{x: A \wedge B, \Gamma \vdash \Delta} L \wedge & \frac{\Gamma \vdash \Delta, x: A \quad \Gamma \vdash \Delta, x: B}{\Gamma \vdash \Delta, x: A \wedge B} R \wedge \\
\frac{x: A, \Gamma \vdash \Delta \quad x: B, \Gamma \vdash \Delta}{x: A \vee B, \Gamma \vdash \Delta} L \vee & \frac{\Gamma \vdash \Delta, x: A, x: B}{\Gamma \vdash \Delta, x: A \vee B} R \vee \\
\frac{\Gamma \vdash \Delta, x: A \quad x: B, \Gamma \vdash \Delta}{x: A \supset B, \Gamma \vdash \Delta} L \supset & \frac{x: A, \Gamma \vdash \Delta, x: B}{\Gamma \vdash \Delta, x: A \supset B} R \supset \\
\frac{x: \perp, \Gamma \vdash \Delta}{L} & L \perp
\end{array}
$$

$$
\begin{array}{lc}
\frac{y: A, x: \square A, x R y, \Gamma \vdash \Delta}{x: \square A, x R y, \Gamma \vdash \Delta} & L \square \quad \frac{x R y, \Gamma \vdash \Delta, y: A}{\Gamma \vdash \Delta, x: \square A} R \square \\
\frac{x R y, y: A, \Gamma \vdash \Delta}{x: \diamond A, \Gamma \vdash \Delta} L \diamond & \frac{x R y, \Gamma \vdash \Delta, x: \diamond A, y: A}{x R y, \Gamma \vdash \Delta, x: \diamond A} R \diamond
\end{array}
$$

In $R \square$ and $L \diamond, y$ does not occur in the conclusion.

Fig. 1. G3K: a labeled proof system for the modal logic $K$

$$
\begin{gathered}
\frac{x R x, \Gamma \vdash \Delta}{\Gamma \vdash \Delta} \text { refl } \frac{x R z, x R y, y R z, \Gamma \vdash \Delta}{x R y, y R z, \Gamma \vdash \Delta} \text { trans } \frac{y R x, x R y, \Gamma \vdash \Delta}{x R y, \Gamma \vdash \Delta} \text { symm } \\
\frac{y R z, x R y, x R z, \Gamma \vdash \Delta}{x R y, x R z, \Gamma \vdash \Delta} \text { eucl } \quad \frac{x R y, \Gamma \vdash \Delta}{\Gamma \vdash \Delta} \operatorname{ser} \quad \frac{y R u, z R u, x R y, x R z, \Gamma \vdash \Delta}{x R y, x R z, \Gamma \vdash \Delta} \operatorname{dir} \\
\frac{x R y, x R z, y R z, \Gamma \vdash \Delta \quad x R y, x R z, z R y, \Gamma \vdash \Delta}{x R y, x R z, \Gamma \vdash \Delta} \operatorname{conn}
\end{gathered}
$$

In ser, $y \notin \Gamma, \Delta$. In $\operatorname{dir}, u$ does not occur in the conclusion.

Fig. 2. Rules for capturing relational properties.

adding such rules to the base system $G 3 K$, we get a system for the corresponding logic. In the following, we will denote by $G 3 K^{*}$ any extension of $G 3 K$ with rules following the geometric rule scheme. We remark that all structural rules (cut included) are admissible in $G 3 K^{*}$ [14].

\subsection{The standard translation from modal logic into classical logic}

The following standard translation (see, e.g., [2]) provides a bridge between propositional modal logic and first-order classical logic:

$$
\begin{aligned}
& S T_{x}(P)=P(x) \\
& S T_{x}(A \supset B)=S T_{x}(A) \supset S T_{x}(B) \\
& S T_{x}(\perp)=\perp \\
& S T_{x}(\square A) \quad=\forall y\left(R(x, y) \supset S T_{y}(A)\right) \\
& S T_{x}(\diamond A) \quad=\exists y\left(R(x, y) \wedge S T_{y}(A)\right)
\end{aligned}
$$

where $x$ is a free variable denoting the world in which the formula is evaluated. The first-order language into which modal formulas are translated is usually referred to as first-order correspondence language [2] and consists of a binary predicate symbol $R$ and a unary predicate symbol $P$ for each $P \in \mathcal{P}$. When a modal operator is translated, a new fresh variable ${ }^{2}$ is introduced. It is easy to

${ }^{2}$ In fact, it is possible to show that every modal formula can be translated into a formula in the fragment of first-order logic which uses only two variables [2]. By the 
show that for any modal formula $A$, any model $\mathcal{M}$ and any world $w$, we have that $\mathcal{M}, w \models A$ if and only if $\mathcal{M}=S T_{x}(A)[x \leftarrow w]$.

\subsection{A focused proof system for first-order classical logic}

Figure 3 presents the LKF proof system from [10] (where some inference rules have been renamed). This system involves polarized formulas, built using atomic formulas, the usual first-order quantifiers $\forall$ and $\exists$, and polarized versions of the logical connectives and constants $t^{-}, t^{+}, f^{-}, f^{+}, \vee^{-}, \vee^{+}, \wedge^{-}$, and $\wedge^{+}$. The positive and negative versions of connectives and constants have identical truth conditions but different inference rules inside the polarized proof system. For example, the introduction rule for $V^{-}$is invertible while the introduction rule for $\mathrm{V}^{+}$is not. All polarized formulas are (as the name implies) either positive or negative. In particular, if a formula's top-level connective is $t^{+}, f^{+}, \mathrm{V}^{+}, \wedge^{+}$, or $\exists$, then that formula is positive. Dually, if a formula's top-level connective is $t^{-}, f^{-}, \vee^{-}, \wedge^{-}$, or $\forall$, then it is negative. In this way, every polarized formula is classified except for literals: to polarize them, we are allowed to fix the polarity of atomic formulas in any way we see fit. We may ask that all atomic formulas are positive, that they are all negative, or we can mix polarity assignments. In any case, if $A$ is a positive atomic formula, then it is a positive formula and $\neg A$ is a negative formula: conversely, if $A$ is a negative atomic formula, then it is a negative formula and $\neg A$ is a positive formula.

We shall find it important to break a sequence of negative or positive connectives by inserting delays: if $B$ is a polarized formula then we define $\partial^{-}(B)$ to be (the always negative) $B \wedge^{-} t^{-}$and $\partial^{+}(B)$ to be (the always positive) $B \wedge^{+} t^{+}$. From such a definition, the following rules can be derived:

$$
\frac{\vdash \Theta \Uparrow B, \Gamma}{\vdash \Theta \Uparrow \partial^{-}(B), \Gamma} \partial^{-} \quad \frac{\vdash \Theta \Downarrow B}{\vdash \Theta \Downarrow \partial^{+}(B)} \partial^{+}
$$

Returning to the proof system in Figure 3, we note that the inference rules there involve two kinds of sequents: $\vdash \Theta \Uparrow \Gamma$ and $\vdash \Theta \Downarrow B$, where $\Theta$ is a multiset of polarized formulas, $B$ is a polarized formula, and $\Gamma$ is a list of polarized formulas. (It is possible to relax the list structure of $\Gamma$ to be a multiset but that relaxation is not useful in this paper.) The formula occurrence $B$ in the $\Downarrow$ sequent is called the focus of that sequent. The completeness of $L K F$ can be stated as follows [10]: if $B$ is an (unpolarized) classical logic theorem and $\hat{B}$ is any polarization of $B$, then $\vdash \cdot \Uparrow \hat{B}$ is provable in $L K F$. Clearly, the choice of polarization does not affect provability but it can have a big impact on the structure of proofs.

To illustrate the use of delays, notice that the sequent $\vdash \Theta \Downarrow \exists x \exists y . B(x, y)$ must be the result of applying (at least) two $\exists$-introduction rules. In contrast, the sequent $\vdash \Theta \Downarrow \exists x \partial^{-}(\exists y . B(x, y))$ must be the conclusion of only one $\exists$-introduction rule: a separate instantiation of $\exists y$ can take place elsewhere in the proof.

decidability of such a fragment, an easy proof of the decidability of propositional modal logic follows. 
ASYNCHRONOUS INTRODUCTION RULES

$$
\begin{aligned}
& \frac{\vdash \uparrow \Uparrow t^{-}, \Gamma}{\vdash-} t^{-} \frac{\vdash \Theta \Uparrow A, \Gamma \quad \vdash \Theta \Uparrow B, \Gamma}{\vdash \Theta \Uparrow A \wedge^{-} B, \Gamma} \wedge^{-} \frac{\vdash \Theta \Uparrow \Gamma}{\vdash \Theta \Uparrow f^{-}, \Gamma} f^{-} \frac{\vdash \Theta \Uparrow A, B, \Gamma}{\vdash \Theta \Uparrow A \vee^{-} B, \Gamma} \vee^{-} \\
& \frac{\vdash \Theta \Uparrow[y / x] B, \Gamma}{\vdash \Theta \Uparrow \forall x . B, \Gamma} \forall \dagger
\end{aligned}
$$

SYNCHRONOUS INTRODUCTION RULES

$$
\frac{\vdash}{\vdash \Theta \Downarrow t^{+}} t^{+} \frac{\vdash \Theta \Downarrow B_{1} \vdash \Theta \Downarrow B_{2}}{\vdash \Theta \Downarrow B_{1} \wedge^{+} B_{2}} \wedge^{+} \frac{\vdash \Theta \Downarrow B_{i}}{\vdash \Theta \Downarrow B_{1} \vee^{+} B_{2}} \vee^{+}, i \in\{1,2\} \quad \frac{\vdash \Theta \Downarrow[t / x] B}{\vdash \Theta \Downarrow \exists x . B} \exists
$$

IDENTITY RULES

$$
\frac{\vdash \neg P_{a}, \Theta \Downarrow P_{a}}{\vdash \text { init }} \frac{\vdash \Theta \Uparrow B \quad \vdash \Theta \Uparrow \neg B}{\vdash \Theta \Uparrow \cdot} \text { cut }
$$

Structural RULes

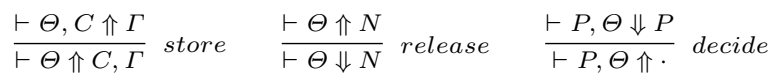

Here, $P$ is a positive formula; $N$ a negative formula; $P_{a}$ a positive literal; $C$ a positive formula or negative literal; and $\neg B$ is the negation normal form of the negation of $B$. The proviso marked as $\dagger$ is the usual eigenvariable restriction: $y$ is not free in $\Theta$, in $\Gamma$, nor in $\forall x$. $B$.

Fig. 3. The $L K F$ focused proof systems for classical logic (minor differences from [10]).

A polarized formula $B$ is a bipolar formula if $B$ is a positive formula and no positive subformula occurrence of $B$ is in the scope of a negative connective in $B$. A bipole is a pair of a negative phase below a positive phase within LKF: thus, bipoles are macro inference rules in which the conclusion and the premises are 介-sequents with no formulas to the right of the up-arrow.

\section{$3 \quad$ Labeled proof systems and focused proof systems}

In this section, we compare derivations in G3K (and its extensions) and $L K F$. In particular, we show that there is a strict correspondence between rule applications in the former and bipoles in the latter. In order to do that, we will define a translation from labeled modal formulas into first-order polarized formulas.

\subsection{From labeled modal formulas to polarized first-order formulas}

Note that the set of connectives used in the language of $G 3 K$ differs from that of $L K F$, where formulas are assumed to be in negation normal form. Given a modal formula $A$, we denote with $A^{\circ}$ its negation normal form. In our translation into polarized formulas, we sometimes put a delay in front of a formula only if it is not a literal. For that purpose, we define $A^{\partial^{+}}$(when $A$ is a first-order formula in negation normal form) to be $A$ if $A$ is a literal and $\partial^{+}(A)$ otherwise. We extend such a notion to a multiset $\Gamma$ of formulas by defining $\Gamma^{\partial^{+}}=\left\{A^{\partial^{+}} \mid A \in \Gamma\right\}$.

We are now in a position to present our translation from the language of $G 3 K$ into the language of $L K F$. Such a translation is based on the standard translation recalled in Section 2. Given a world $x$, we define the translation [. $]_{x}$ from modal formulas in negation normal form into polarized first-order formulas as: 


$$
\begin{aligned}
& {[P]_{x}=P(x) \quad\left[A^{\circ} \wedge B^{\circ}\right]_{x}=\left[A^{\circ}\right]_{x}{ }^{\partial^{+}} \wedge^{-}\left[B^{\circ}\right]_{x}{ }^{\partial^{+}}} \\
& {[\neg P]_{x}=\neg P(x) \quad\left[A^{\circ} \vee B^{\circ}\right]_{x}=\left[A^{\circ}\right]_{x}{ }^{\partial^{+}} \vee^{-}\left[B^{\circ}\right]_{x} \partial^{+}} \\
& {[\perp]_{x}=f^{-} \quad\left[\diamond A^{\circ}\right]_{x} \quad=\exists y\left(R(x, y) \wedge^{+} \partial^{-}\left(\left[A^{\circ}\right]_{y}{ }^{{ }^{+}}\right)\right)} \\
& {\left[\square A^{\circ}\right]_{x}=\forall y\left(\neg R(x, y) \vee^{-}\left[A^{\circ}\right]_{y}{ }^{\partial^{+}}\right)}
\end{aligned}
$$

Based on this, we define the translation [.] from labeled formulas and relational atoms into polarized first-order formulas as $[x: A]=\left[A^{\circ}\right]_{x}$ and $[x R y]=R(x, y)$. In the following, we will sometimes use the natural extension of this notion to multisets of labeled formulas, i.e., $[\Gamma]=\{[\varphi] \mid \varphi \in \Gamma\}$. Note that predicates of the form $P(x)$ and $R(x, y)$ are considered as having positive polarity.

Finally, we define a translation from $G 3 K$ sequents into $L K F$ sequents:

$$
\left[\left(\varphi_{1}, \ldots, \varphi_{n} \vdash \psi_{1}, \ldots, \psi_{m}\right)\right]=\vdash\left[\neg \varphi_{1}\right]^{\partial^{+}}, \ldots,\left[\neg \varphi_{n}\right]^{\partial^{+}},\left[\psi_{1}\right]^{\partial^{+}}, \ldots,\left[\psi_{m}\right]^{\partial^{+}} \Uparrow \cdot
$$

where $[\neg \varphi]$ is $\left[(\neg A)^{\circ}\right]_{x}$ if $\varphi=x: A$ and is $\neg R(x, y)$ if $\varphi=x R y$. We will sometimes write $\neg \Gamma$ to denote $\{\neg A \mid A \in \Gamma\}$.

\subsection{From G3K to $L K F$}

Given two multisets of $L K F$ formulas $\Gamma$ and $\Gamma^{\prime}$, we say that $\Gamma^{\prime}$ extends $\Gamma$ if $\Gamma^{\prime}$ contains $\Gamma$ and $F V(\Gamma)=F V\left(\Gamma^{\prime}\right)$, where $F V(\Delta)$ denotes the set of free variables occurring free in $\Delta$. We say that an $L K F$ sequent $\vdash \Gamma \Uparrow \cdot$ extends an $L K F$ sequent $\vdash \Gamma^{\prime} \Uparrow \cdot$ if $\Gamma$ extends $\Gamma^{\prime}$.

Lemma 1. Let $\frac{S_{1}}{S} r\left(\frac{S_{1} S_{2}}{S} r\right)$ be an application of a rule in G3K. Then for any LKF sequent $S^{\prime}$ that extends $[S]$, there exists a derivation $\left.\begin{array}{c}S_{1}^{\prime} \\ \vdots\end{array} \quad \begin{array}{cc}S_{1}^{\prime} & S_{2}^{\prime} \\ \vdots & S^{\prime}\end{array}\right)$ in LKF, which is a bipole, and such that $S_{1}^{\prime}$ extends $\left[S_{1}\right]\left(S_{1}^{\prime}\right.$ and $\underset{S_{2}^{\prime}}{S^{\prime \prime}}$ extend $\left[S_{1}\right]$ and $\left[S_{2}\right]$, respectively). Furthermore, if $\bar{S}^{r}$ is a rule application in $G 3 K$, then for any $L K F$ sequent $S^{\prime}$ that extends $[S]$, there exists a proof of $S^{\prime}$ that is a bipole.

Proof. The proof proceeds by considering all the rules of G3K. For example, the translation of the $R \square$ from Figure 1 is given by following derivation in $L K F$ :

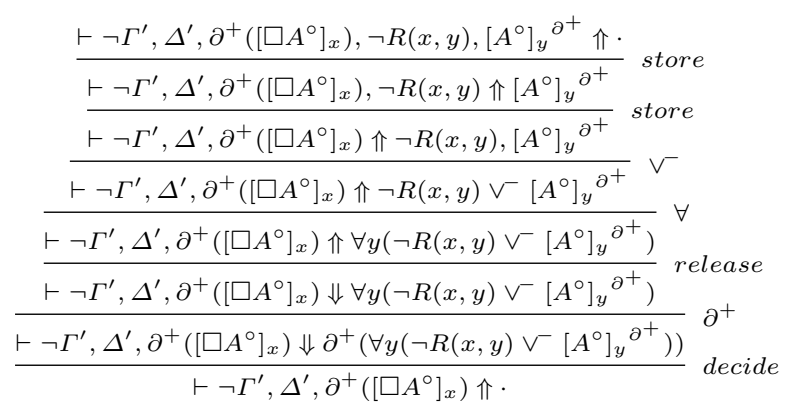

Here $\Gamma^{\prime}$ is any extension of $[\Gamma]^{\partial^{+}}$and $\Delta^{\prime}$ is any extension of $[\Delta]^{\partial^{+}}$. Note that the condition on free variables in the definition of extension ensures that $\forall$ can be applied in the derivation above, as the constraint on eigenvariables is satisfied. 
Theorem 2. Let $\Pi$ be a G3K derivation of a sequent $S$ from the sequents $S_{1}, \ldots, S_{n}$. Then there exists an $L K F$ derivation $\Pi^{\prime}$ of $[S]$ from $\left[S_{1}\right], \ldots,\left[S_{n}\right]$ (such that each rule application in $\Pi$ corresponds to a bipole in $\Pi^{\prime}$ ).

Proof. We proceed bottom-up by starting from the root of $\Pi$ and build $\Pi^{\prime}$ by repeatedly applying Lemma 1 . At each step, we get leaves that are extensions of the ones in $\Pi$, so that Lemma 1 can be applied again.

\subsection{From $L K F$ to G3K}

Given two multisets of $L K F$ formulas $\Gamma$ and $\Gamma^{\prime}$, we say that $\Gamma^{\prime}$ is a contraction of $\Gamma$ if $\Gamma$ contains $\Gamma^{\prime}$ and for each formula $A$ in $\Gamma$ there is at least one occurrence of $A$ in $\Gamma^{\prime}$, i.e., $\Gamma$ and $\Gamma^{\prime}$ contain the same set of formulas but $\Gamma$ can have more occurrences. We say that an $L K F$ sequent $\vdash \Gamma^{\prime} \Uparrow \cdot$ is a contraction of an LKF sequent $\vdash \Gamma \Uparrow \cdot$ if $\Gamma^{\prime}$ is a contraction of $\Gamma$.

Lemma 3. Let $S^{\prime}$ be an LKF sequent of the form $\vdash \Gamma^{\prime} \Uparrow \cdot$ such that each formula in $S^{\prime}$ is the translation $[\varphi]$ of some G3K formula $\varphi$. For each derivation of the form $\begin{gathered}S_{1}^{\prime} \\ \vdots \\ S^{\prime}\end{gathered}\left(\begin{array}{cc}S_{1}^{\prime} & S_{2}^{\prime} \\ \vdots & \\ & S^{\prime}\end{array}\right)$ in LKF that is a bipole, there exist:

(i) a G3K sequent $S$, such that $S^{\prime}$ is a contraction of $[S]$; and

(ii) a rule application $\frac{S_{1}}{S}\left(\frac{S_{1} S_{2}}{S}\right)$ in $G 3 K$ such that $S_{1}^{\prime}=\left[S_{1}\right]\left(S_{1}^{\prime}=\left[S_{1}\right]\right.$ and $\left.S_{2}^{\prime}=\left[S_{2}\right]\right)$.

Furthermore, for each proof of $S^{\prime}$ that is a bipole, there exist a G3K sequent $S$, such that $S^{\prime}$ is a contraction of $[S]$, and a rule application $\bar{S}$ init in G3K.

Proof. We have one case for each possible G3K formula $\varphi$ on the translation of which a decide is applied. Let us consider one representative case.

Let $\varphi=x: \diamond A$. Then we have the following derivation in LKF:

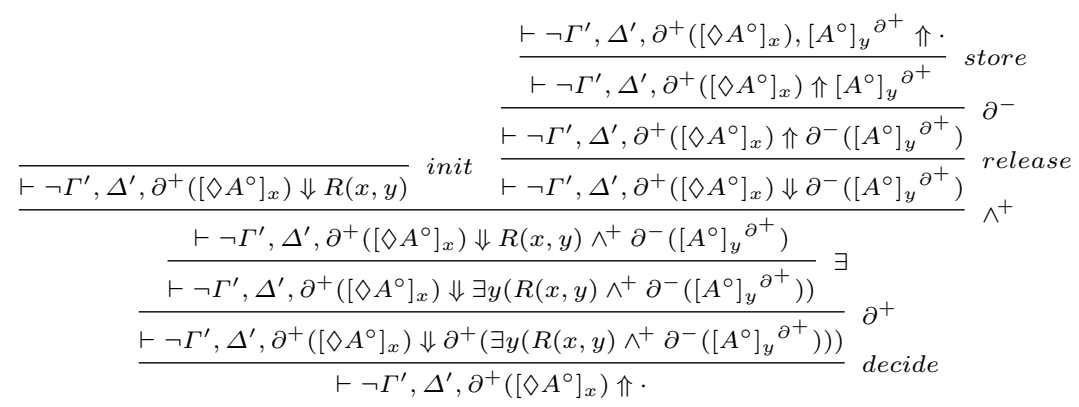

Note that in order to be able to apply the rule init, and thus have indeed a bipole, the multiset $\neg \Gamma^{\prime}$ must contain the formula $\neg R(x, y)$. But then, in G3K, we can have the following corresponding rule application:

$$
\frac{x R y, \Gamma \vdash \Delta, x: \diamond A, y: A}{x R y, \Gamma \vdash \Delta, x: \diamond A} R \diamond
$$


where $\Gamma^{\prime}=[\Gamma]^{\partial^{+}}$and $\Delta^{\prime}=[\Delta]^{\partial^{+}}$.

Theorem 4. Let $\Pi^{\prime}$ be a proof of a sequent $S^{\prime}$ in LKF such that $S^{\prime}=[S]$ for some G3K-sequent $S$. Then there exists a proof $\Pi$ of $S$ in $G 3 K$ (such that each bipole in $\Pi^{\prime}$ corresponds to a single rule application in $\left.\Pi\right)$.

Proof. We proceed top-down starting from the leaves of $\Pi^{\prime}$ and build $\Pi$ by repeatedly applying Lemma 3 . At each step, we get as the conclusion of a G3K rule application a sequent $S^{*}$ such that the one obtained in the corresponding step of $\Pi^{\prime}$ is a contraction of $\left[S^{*}\right]$. By observing that the contraction rule is (height-preserving) admissible in G3K, we can transform the G3K derivation built so far in order to remove possible undesired multiple occurrences of a formula.

The strong correspondence between labeled rule applications and $L K F$ bipoles can also be used to get an immediate proof of the completeness of G3K.

Corollary 5. The system G3K is complete.

Proof. Follows from the completeness of LKF, the adequacy of the standard translation from the modal language into the first-order classical language (on which our translation is based) and Theorem 4.

\subsection{Extensions of $\mathrm{K}$}

Here we show how the results of the previous section can be extended to modal logics whose Kripke frames are characterized by properties expressible by means of geometric axioms.

We recall from Section 2 that a geometric axiom has the form:

$$
\forall \bar{z}\left(P_{1} \wedge \ldots \wedge P_{m} \supset\left(\exists x_{1}\left(Q_{11} \wedge \ldots \wedge Q_{1 k_{1}}\right) \vee \ldots \vee \exists x_{n}\left(Q_{n 1} \wedge \ldots \wedge Q_{n k_{n}}\right)\right)\right)
$$

In $L K F$, we can consider geometric extensions of the logic $K$ by adding the corresponding geometric axioms in the left-side of the sequent to be derived. We propose the following translation, involving polarization of connectives, for axioms $G$ having the form shown above ${ }^{3}$ :

$\exists \bar{z}\left(\left(P_{1} \wedge^{+} \ldots \wedge^{+} P_{m}\right) \wedge^{+}\left(\forall x_{1}\left(\neg Q_{11} \vee^{-} \ldots \vee^{-} \neg Q_{1 k_{1}}\right) \wedge^{-} \ldots \wedge^{-} \forall x_{n}\left(\neg Q_{n 1} \vee^{-} \ldots \vee^{-} \neg Q_{n k_{n}}\right)\right)\right)$

As recalled in Section 2, the system G3K can be extended to capture all the modal logics characterized by geometric axioms, by modularly adding to the base system rules defined according to a proper scheme [14]. Each application of such a rule corresponds to a single bipole in $L K F$, as shown in Figure 4. This fact ensures that the statements of Theorems 2 and 4 (as well as Corollary 5) hold also for any geometric extension of $K$ and any system $G 3 K^{*}$.

\footnotetext{
${ }^{3}$ Note that in $L K F$ we consider one-sided sequents and the one we propose is in fact a polarization of the negation of the axiom.
} 


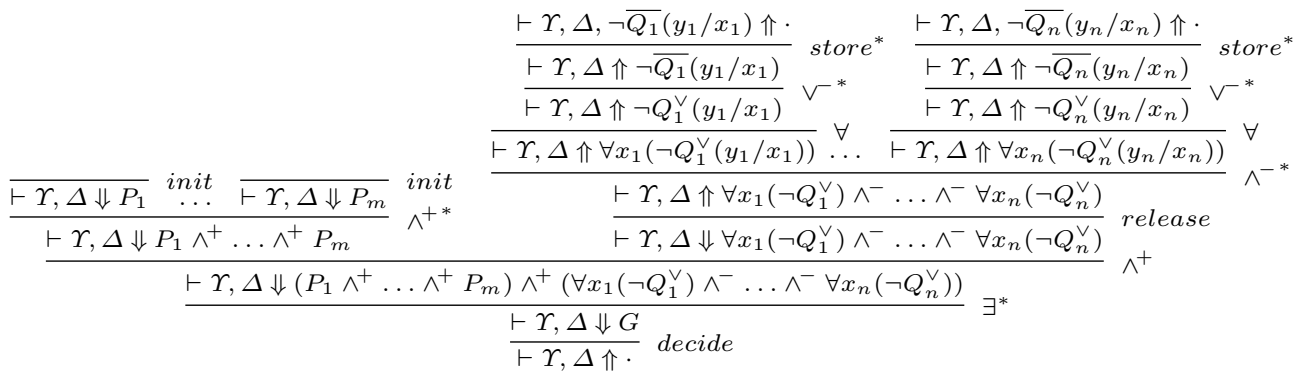

where $\Upsilon$ is an abbreviation for $G, \neg \bar{P}, \neg \Gamma$ and $\neg Q_{i}^{\vee}$ stands for $\neg Q_{i 1} \vee \ldots \vee \neg Q_{i k_{i}}, \neg \overline{Q_{i}}$ stands for $\neg Q_{i 1}, \ldots, \neg Q_{i k_{i}}$ and in ${ }^{*}$, for simplicity, we have applied several instances of the same rule in a single step.

Fig. 4. $L K F$ derivation (bipole) corresponding to a geometric rule application in $G 3 K^{*}$.

\subsection{Checking G3K proofs via $L K F$}

The results in this section can be immediately applied to the ProofCert project [4, 11], where a general proof checker, based on $L K F$ (or on its intuitionistic version $L J F)$ and implemented in $\lambda$ Prolog [12], is used to check proofs in a wide range of formats. To this aim, $L K F$ is augmented as follows [3]: $(i)$ a proof certificate term, which describes the proof evidence to be checked, is added to every sequent; $(\mathrm{ii})$ every inference rule of $L K F$ has an additional premise using either an expert or a clerk predicate; and ( iii) formulas to the left of $\Uparrow$ and $\Downarrow$ are now associated to an index, used to regulate the mechanism of storing and deciding.

A foundational proof certificate for a given proof format consists in the definition of a translation of formulas from the original language into the language of $L K F$ and in the definition of expert and clerk predicates. Expert predicates are used to drive the checking process during the synchronous phase (e.g., by specifying which term has to be used to instantiate an existential), while clerk predicates are used in the asynchronous phase not to extract information from the certificates but only to do routine computations. (The full augmented system $L K F^{a}$ is available in the extended version of this paper.) To illustrate the idea, we show here the augmented version of the decide rule:

$$
\frac{\Xi^{\prime} \vdash \Theta \Downarrow P \quad \operatorname{decide}_{e}\left(\Xi, \Xi^{\prime}, l\right) \quad\langle l, P\rangle \in \Theta \quad \text { positive }(P)}{\Xi \vdash \Theta \Uparrow \cdot} \text { decide }^{a}
$$

Here $\Xi$ and $\Xi^{\prime}$ are certificates, $l$ is the index specifying the formula on which to decide and the expert predicate decide $_{e}$ extracts $\Xi^{\prime}$ and $l$ from $\Xi$.

By using the encoding proposed in this section and by exploiting the shown correspondence between inference rules and bipoles, $G 3 K$ proof evidence can be described (and then checked in $L K F^{a}$ ) by using very simple proof certificates, basically consisting in the sequence of (translated and polarized) formulas on which to decide. The expert for the decide rule simply decides on the formula on top of the certificate and removes it from the certificate itself. A simple version 
of it can have the following $\lambda$ Prolog form:

$$
\forall A \forall \Gamma \text {. } \operatorname{decide}_{e}([A \mid \Gamma], \Gamma, A) .
$$

where we use the formula $A$ itself as an index. The other clerk and expert predicates are either empty or only used to "propagate" information. ${ }^{4}$

\section{Focused labeled proof systems for modal logic}

\subsection{A focused system for the logic $K$}

We have shown how the standard translation from modal logic into classical logic, when enriched with a proper polarization, allows one to define a strong correspondence between labeled modal derivations and focused classical derivations. Here we go further and define a focused labeled system $(L M F)$ for modal logic.

The basic idea is to define a restriction of $L K F$ targeting the formulas arising from the modal language, i.e., such that the quantifier rules are only applied to formulas that represent the translation of modal formulas of the form $\square A$ or $\diamond A . L M F$ is defined over a labeled modal language: formulas have the form $x: A, x R y$ or $\neg x R y$, where $A$ is a polarized modal formula in negation normal form. Note that here, differently from $G 3 K$, since we consider one-sided sequents, relational atoms can also occur negated. The following $L K F$ derivations show the way we extract the modal rules, from $\forall$ and $\exists$, by restricting the use of the first-order quantifiers to only express (a proper polarization of) the standard translation of formulas having $\square$ or $\diamond$ as the main connective:

$$
\begin{array}{ll}
\frac{\vdash \Theta, \neg R(x, y) \Uparrow A(y), \Gamma}{\vdash \Theta \Uparrow \neg R(x, y), A(y), \Gamma} \text { store } & \frac{\vdash \Theta \Downarrow R(x, y) \vdash \Theta \Downarrow A(y)}{\vdash \Theta \Downarrow R(x, y) \wedge^{+} A(y)} \wedge^{+} \\
\frac{\vdash \Theta \Uparrow \neg R(x, y) \vee^{-} A(y), \Gamma}{\vdash \Theta \Uparrow \forall y\left(\neg R(x, y) \vee^{-} A(y)\right), \Gamma} \forall & \frac{1}{\vdash \Theta \Downarrow \exists y\left(R(x, y) \wedge^{+} A(y)\right)} \exists
\end{array}
$$

From such derivations, we get the following rules $\square_{K}$ and $\diamond_{K}$, respectively:

$$
\frac{\vdash \Theta, \neg x R y \Uparrow y: B, \Gamma}{\vdash \Theta \Uparrow x: \square B, \Gamma} \square_{K} \frac{\vdash \Theta \Downarrow x R y \quad \vdash \Theta \Downarrow y: B}{\vdash \Theta \Downarrow x: \diamond B} \diamond_{K}
$$

where, in analogy with the side-condition on the $\forall$ rule, we have that in $\square_{K}, y$ is not free in $\Theta$ nor in $\Gamma$. The whole system $L M F$ is presented in Figure 5.

The notion of polarizing an (unpolarized) propositional modal formula in the $L M F$ setting is essentially the same as it was in the $L K F$ setting. In particular, the polarities of $\diamond B$ and $\square B$ are, respectively, positive and negative. The propositional constants and the conjunction and disjunction are ambiguous and can be made either positive or negative. Finally, the atomic formulas that result from

\footnotetext{
${ }^{4}$ We note that in this way, we provide no information on which substitution term to use in case of existential quantifiers, and let such terms be reconstructed by the checker. In order to obtain a completely faithful encoding of the original G3K proof, the label term used for instantiating $\diamond$-formulas should also be contained in the proof certificate and the expert predicate for the $\exists$ should take that into account.
} 
ASYNCHRONOUS INTRODUCTION RULES

$$
\begin{gathered}
\frac{\vdash \Theta \Uparrow x: t^{-}, \Gamma}{\vdash t^{-}} \frac{\vdash \Theta \Uparrow \Gamma}{\vdash \Theta \Uparrow x: f^{-}, \Gamma} f^{-} \\
\frac{\vdash \Theta \Uparrow x: A, \Gamma \quad \vdash \Theta \Uparrow x: B, \Gamma}{\vdash \Theta \Uparrow x: A \wedge^{-} B, \Gamma} \wedge_{K}^{-} \frac{\vdash \Theta \Uparrow x: A, x: B, \Gamma}{\vdash \Theta \Uparrow x: A \vee^{-} B, \Gamma} \vee_{K}^{-} \frac{\vdash \Theta, \neg x R y \Uparrow y: B, \Gamma}{\vdash \Theta \Uparrow x: \square B, \Gamma} \square_{K}
\end{gathered}
$$

SYNCHRONOUS INTRODUCTION RULES

$$
\begin{gathered}
\frac{\vdash \Theta \Downarrow x: B_{1} \quad \vdash \Theta \Downarrow x: B_{2}}{\vdash \Theta \Downarrow x: t^{+}} t^{+} \wedge_{K}^{+} \\
\frac{\vdash \Theta \Downarrow x: B_{1} \wedge^{+} B_{2}}{\vdash \Theta \Downarrow x: B_{i}} \\
\frac{\vdash \Downarrow \Downarrow x: B_{1} \vee^{+} B_{2}}{\vdash \Theta} \vee_{K}^{+}, i \in\{1,2\} \frac{\vdash \Theta \Downarrow x R y \quad \vdash \Theta \Downarrow y: B}{\vdash \Theta \Downarrow x: \diamond B} \diamond_{K}
\end{gathered}
$$

IDENTITY RULES

$$
\frac{}{\vdash x: \neg P_{a}, \Theta \Downarrow x: P_{a}} \text { init }_{K} \quad \frac{\vdash \neg x R y, \Theta \Downarrow x R y}{\vdash-\text { nit }_{R K}} \frac{\vdash \Theta x: B \quad \vdash \Theta \Uparrow x: \neg B}{\vdash \Theta \Uparrow \cdot} \text { cut }_{K}
$$

STRUCTURAL RULES

$$
\frac{\vdash \Theta, x: C \Uparrow \Gamma}{\vdash \Theta \Uparrow x: C, \Gamma} \text { store }_{K} \quad \frac{\vdash \Theta \Uparrow x: N}{\vdash \Theta \Downarrow x: N} \text { release }_{K} \frac{\vdash x: P, \Theta \Downarrow x: P}{\vdash x: P, \Theta \Uparrow \cdot} \text { decide }_{K}
$$

Here, $x: P$ is a positive formula; $x: N$ a negative formula; $x: P_{a}$ and $x R y$ positive literals; $x: C$ a positive formula or negative literal; and $\neg B$ is the negation normal form of the negation of $B$. In $\square_{K}, y$ is not free in $\Theta$ nor in $\Gamma$.

Fig. 5. $L M F$ : a focused labeled proof system for the modal logic $K$

translating propositional constants in modal formulas are also ambiguous and can be given any arbitrary but fixed polarization. In the case of $L M F$, we shall fix the polarity of relational atoms to be positive. This latter choice is important for us in the next section to properly describe the geometric-axioms-as-inference-rules.

Theorem 6. The system LMF is sound and complete with respect to the logic $K$, for any polarization of formulas.

Proof. If we read a labeled formula $x: A$ as the first-order formula $A(x)$ and a relational atom $x R y$ as $R(x, y)$, we have that $L M F$ is just obtained by $L K F$ by replacing the rules $\forall$ and $\exists$ by $\square_{K}$ and $\nabla_{K}$, respectively (note that the rule init is just split into init $_{K}$ and init $\left._{R K}\right)$. We have shown that $\square_{K}$ and $\nabla_{K}$ are simply restrictions of $\forall$ and $\exists$ to the first-order translation of modalities. Soundness and completeness of LMF then easily follow from soundness and completeness, with respect to any polarization of formulas, of LKF (see Section 2) and by the adequacy of the standard translation from propositional modal logic into first-order classical logic.

As it was the case for linear, classical and intuitionistic logic, this focused system allows for obtaining a normal form of cut-free modal derivations. We can illustrate that with a simple example: a proof of the labeled formula $x: \diamond \diamond \neg P \vee$ $\square \square P$ can have several forms in G3K, as we can alternate applications of $R \square$ and $R \diamond$, while there is only one cut-free proof of its polarization $x: \diamond \diamond \neg P \vee \square \square P$ in $L M F$, which first applies (in a single phase) all the $\square_{K}$ and then (in another phase) all the $\nabla_{K}$. 


\subsection{Focused systems for extensions of $\mathrm{K}$}

We can extend $L M F$ to a focused system for any geometric extension of $K$ by replacing the first-order axioms with rules manipulating the relational atoms. Namely, given a geometric axiom of the form $\forall \bar{z}\left(P_{1} \wedge \ldots \wedge P_{m} \supset\left(\exists x_{1}\left(Q_{11} \wedge \ldots \wedge\right.\right.\right.$ $\left.\left.\left.Q_{1 k_{1}}\right) \vee \ldots \vee \exists x_{n}\left(Q_{n 1} \wedge \ldots \wedge Q_{n k_{n}}\right)\right)\right)$, we can extract, from an $L K F$ derivation analogous to the one in Figure 4, the following rule scheme:

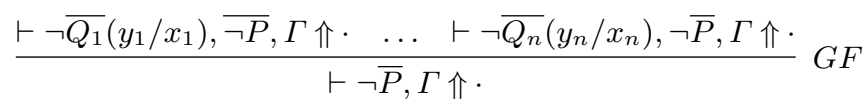

where $\overline{Q_{j}}$ and $\bar{P}$ denote the multisets of relational atoms $Q_{j 1}, \ldots, Q_{j k_{j}}$ and $P_{1}, \ldots, P_{m}$, respectively, and the eigenvariables $y_{1}, \ldots, y_{n}$ do not occur free in the conclusion. Note that in order to build a complete bipole once we focus on the formula representing the geometric axiom (polarized as in Figure 4), the literals $\bar{P}$ must be present in the context. Given a set of geometric frame properties $F_{1}, \ldots, F_{n}$, by adding modularly the corresponding $G F$-based rules to the base system $L M F$, we get a focused labeled system for the logic $K F_{1}, \ldots, F_{n}$. Soundness and completeness of any such extended system directly follows from the way we have derived the rule.

The rule scheme $G F$ comes along the lines of the one given in [14] (see Section 2). We notice that in the more specific case of universal axioms (described and treated in the context of sequent systems in [15]), i.e., axioms of the form $\forall \bar{z}\left(P_{1} \wedge \ldots \wedge P_{m} \supset Q_{1} \vee \ldots \vee Q_{n}\right)$ the following rule scheme could also be used:

$$
\frac{\vdash P_{1}, \bar{Q}, \Gamma \Uparrow \cdot \ldots \vdash P_{m}, \bar{Q}, \Gamma \Uparrow \cdot}{\vdash \bar{Q}, \Gamma \Uparrow \cdot} U F
$$

where $\bar{Q}$ denotes the multiset of relational atoms $Q_{1}, \ldots, Q_{n}$. Such a rule scheme is justified by the following LKF derivation, where the formula on the right-side of the root sequent is a proper polarization of (the negation of) a universal axiom:

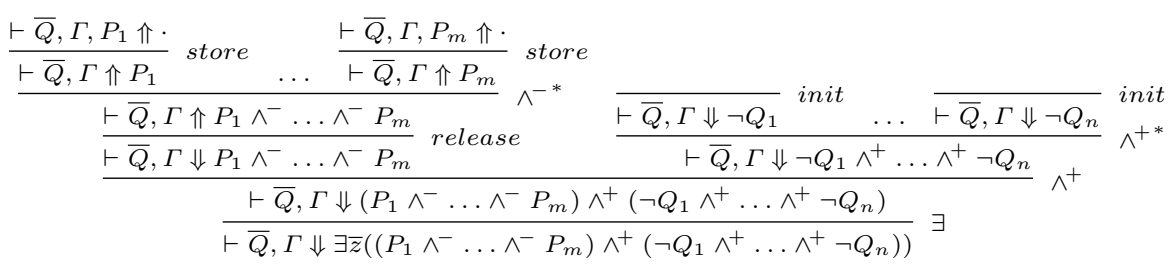

where in ${ }^{*}$, for simplicity, we have applied several instances of the same rule in a single step. Note that, in this case, relational atoms need to be assigned negative polarity (and small adjustments should be made to the base system LMF in order to deal with this fact).

The rule scheme $U F$ cannot be applied in the general case of geometric axioms. In fact, with regard to the derivation shown above, one can notice that in the presence of a geometric axiom, the conjunction of negated $Q_{i}$ atoms would occur under the scope of a universal quantification and we would not be able to process the whole formula inside a single bipole. 
The different formulation of the $G F$ and $U F$ rule schemes seems to be related to two different approaches present in the literature of labeled (natural deduction) systems for modal logics. As an example, we show here two couples of natural deduction rules for expressing the (universal) property of transitivity and the (non-universal) property of seriality:

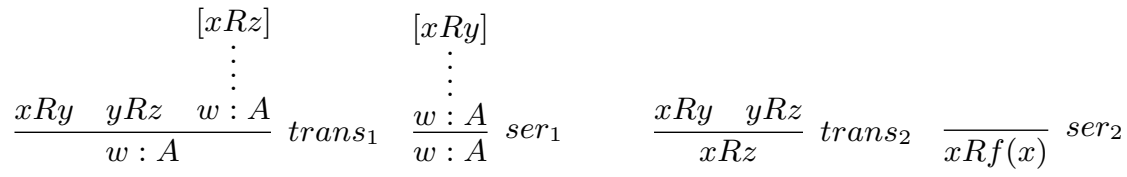

where $y$ is fresh in $\operatorname{ser}_{1}$. The rules trans $_{1}$ and $\operatorname{ser}_{1}$ (from [18], in the style of $G F$ ) follow a pattern that allows capturing all geometric properties and is used in the context of a natural deduction system where no rule has a relational atom in the conclusion. The rules $\operatorname{trans}_{2}$ and $\operatorname{ser}_{2}$ (from [19], in the style of $U F$ ) express relational properties in a more direct fashion (only capturing Horn formulas) where the "freshness" of the variable to be introduced, in the case of a truly geometric axiom, is recovered by using Skolem functions (as in $\operatorname{ser}_{2}$ ).

\section{Conclusion and future work}

The approach to describing modal logic proof systems via labeled sequents provides a way to reduce provability in modal logic to provability in first-order logic. This reduction is also modular: different modal logics can be presented using different theories describing the structure of their Kripke frames. When these theories - taken as assumptions - are also geometric, their use can be understood, instead, as inference rules [14]. Our work here using focused proof systems takes this connection one step further: we are able to faithfully encode the inference rules of labeled modal logic as bipoles in focused proofs in first-order logic. We note that in [16], an encoding of the same labeled modal proof system into a framework based on linear logic with subexponentials was proposed, with the aim of checking meta-properties of the system. The kind of encoding that we propose here is useful for the ProofCert project $[3,11]$, where a general proof checker for focused first-order classical (and intuitionistic) logic is used to check proofs in a wide range of different formats: the encoding in Section 3 is all that is needed for that general purpose proof checker to check labeled sequent calculus proofs of modal logic formulas. We also believe that the simple foundational proof certificate sketched there for labeled modal sequent systems can be easily adapted and extended to work with prefixed tableaux for modal logic [7]. As a next step, we plan to define and implement, in the context of ProofCert, foundational proof certificates for theorem provers based on such an approach.

Another natural future direction is to consider a version of the focused calculus $L M F$ but for intuitionistic modal logic (as, e.g., the ones considered in [18]) by using as a basis (instead of $L K F$ ) the focused proof system $L J F$ for intuitionistic logic [10]. Similarly, this work could be extended to investigate the relationship between focused proof systems and labeled proof systems for other non-classical 
logics $[8,19]$, such as intermediate logics [5]. Another interesting direction consists in considering non-labeled deduction systems for modal logic (see [7] for a general account), and in particular modal proof systems based on generalizations/extensions of sequents, such as hypersequents or nested sequents. In that setting, more sophisticated focusing mechanisms seem to be necessary in order to get an appropriate correspondence between modal inference rules and bipoles.

Acknowledgments. This work was carried out during the tenure of an ERCIM Alain Bensoussan Fellowship Programme by the second author and was funded by the ERC Advanced Grant ProofCert.

\section{References}

1. Jean-Marc Andreoli. Logic programming with focusing proofs in linear logic. J. of Logic and Computation, 2(3):297-347, 1992.

2. Patrick Blackburn and Johan Van Benthem. Modal logic: a Semantic Perspective. In Handbook of Modal Logic, pp 1-82. Elsevier, 2007.

3. Zakaria Chihani, Dale Miller, and Fabien Renaud. Foundational proof certificates in first-order logic. In CADE 24, LNAI 7898, pp. 162-177, 2013.

4. Zakaria Chihani, Tomer Libal, and Giselle Reis. System Description: The Proof Certifier Checkers. To appear in Tableaux 2015.

5. Roy Dyckhoff and Sara Negri. Proof analysis in intermediate logics. Archive for Mathematical Logic, 51(1-2):71-92, 2012.

6. Roy Dyckhoff and Sara Negri. Geometrisation of first-order logic. The Bulletin of Symbolic Logic, 21:123-163, 62015.

7. Melvin Fitting. Modal proof theory. In Frank Wolter Patrick Blackburn, Johan van Benthem, editor, Handbook of Modal Logic, pages 85-138. Elsevier, 2007.

8. Dov M. Gabbay. Labelled Deductive Systems. Clarendon Press, 1996.

9. Jean-Yves Girard. On the meaning of logical rules I: syntax vs. semantics. In Berger and Schwichtenberg, eds, Computational Logic, pp. 215-272. Springer, 1999.

10. Chuck Liang and Dale Miller. Focusing and polarization in linear, intuitionistic, and classical logics. Theor. Comput. Sci., 410(46):4747-4768, 2009.

11. Dale Miller. A proposal for broad spectrum proof certificates. In J.-P. Jouannaud and Z. Shao, editors, CPP 2011, LNCS 7086, pp. 54-69.

12. Dale Miller and Gopalan Nadathur. Programming with Higher-Order Logic. Cambridge University Press, June 2012.

13. Dale Miller and Elaine Pimentel. A formal framework for specifying sequent calculus proof systems. Theoretical Computer Science, 474:98-116, 2013.

14. Sara Negri. Proof analysis in modal logic. J. Philos. Logic, 34(5-6):507-544, 2005.

15. Sara Negri and Jan von Plato. Cut elimination in the presence of axioms. Bulletin of Symbolic Logic, 4(4):418-435, 1998.

16. Vivek Nigam, Elaine Pimentel, and Giselle Reis. An extended framework for specifying and reasoning about proof systems. J. of Logic and Computation, 2014

17. Henrik Sahlqvist. Completeness and correspondence in first and second order semantics for modal logic. In North Holland S. Kanger, editor, Proceedings of the Third Scandinavian Logic Symposium, pages 110-143, 1975.

18. Alex K. Simpson. The Proof Theory and Semantics of Intuitionistic Modal Logic. $\mathrm{PhD}$ thesis, School of Informatics, University of Edinburgh, 1994.

19. Luca Viganò. Labelled Non-Classical Logics. Kluwer Academic Publishers, 2000. 